transfusion was potentially avoidable in favor of other management options. We calculated the mean GBS for the appropriate, potentially avoidable, and clearly avoidable categories yielding 12.8, 12.7, and 10.2 respectively. Mortality occurred in 2 of the 48 cases (4\%). Conclusion: In most instances, emergency physicians are effectively integrating hemoglobin thresholds and clinical status to determine if a patients with NVUGIB and hgb $>70$ require blood products.

Keywords: upper gastro-intestinal bleeds, transfusion, emergency medicine

\section{P118 \\ Effects of system design on laboratory utilization in the emergency department: the case for INR \& aPTT}

D. Tawadrous, MD, T. Skoretz, MD, D. Thompson, MD, S.A. Detombe, PhD, K. Van Aarsen, MSc, Western University, London, ON

Introduction: In the context of a shrinking healthcare budget, poor physician cost awareness, and continued over-utilization of low-value tests in the emergency department, we re-designed our computerized order entry system to reduce the use of coagulation testing. Methods: A hospital-based prospective pre-post analysis following de-bundling of INRPTT testing in two academic hospital emergency departments (annual visits 140,000). All participants aged 18 years or older undergoing evaluation and/or treatment at either of during the period of August 1, 2015 to July 24, 2016 were included. Primary outcome is coagulation testing utilization rates and associated costs. Results: Unbundling INR and aPTT testing resulted in significantly decreased bundled INRPTT testing relative to baseline (INRPTT tests per patient per day: 0.60 [95\% CI: $0.57-0.62]$ vs. 0.98 [95\% CI: 0.98-0.99], $\mathrm{p}=0.000$ ), with significantly increased targeted testing (INR tests per patient per day: 0.39 [95\% CI: $0.37-0.42]$ vs. 0.00 [95\% CI: $0.00-0.01], \mathrm{p}=0.000$; PTT tests per patient per day: 0.33 [95\% CI: $0.30-$ 0.36 ] vs. 0.01 [ $95 \% \mathrm{CI}: 0.00-0.01], \mathrm{p}=0.000$ ). As a result of unbundling, there was a significant decrease in costs associated with coagulation testing relative to baseline (Cost per day: \$958.52 [INRPTT \$592.78+ INR $\$ 183.91+$ PTT $\$ 181.83]$ vs. $\$ 1,074.50$ [INRPTT $\$ 1,069.76+$ INR $\$ 2.06+$ PTT $\$ 2.68], \mathrm{p}=0.000$ ), realizing estimated daily and yearly savings of $\$ 115.98$ and $\$ 42,332.70$, respectively. Conclusion: Compared to baseline practice patterns, unbundling coagulation testing resulted in the reduction of coagulation testing suggesting system design and user workflows to be an integral factor to provider practice patterns. Given the significant cost-savings, we recommend institutions carefully re-evaluate their system design and user workflows to optimize emergency department laboratory utilization.

Keywords: laboratory medicine, efficiency, cost analysis

\section{P119}

Health care utilization by patients presenting to the emergency department with mental health complaints

C. Thompson, MSc, S.L. McLeod, MSc, A. Sandre, BSc, B. Borgundvaag, MD, Schwartz/Reisman Emergency Medicine Institute, Toronto, ON

Introduction: Emergency department (ED) visits for mental health and addiction related complaints are common and appear to be increasing. It is believed these patients come to the ED requiring urgent assessment either because they do not have a primary care or psychiatric healthcare provider or access to their provider is not available in a timely fashion. The objective of this study was to describe healthcare utilization in the previous 12 months by patients presenting to the ED with a mental health complaint. Methods: Between April-November 2016, a convenience sample of adult ( $\geq 18$ years) patients presenting to an academic ED (annual census 65,000) with a mental health and/or addictions complaint were invited to complete a paper-based survey to determine their usage of ten different mental healthcare resources over the previous 12 months. The questionnaire was pilot-tested and peerreviewed for feasibility and comprehension. Results: Of the 134 patients who completed the survey, mean (SD) age was 37.9 (15.7) years and $64(47.8 \%)$ were male. Only 7 (5.2\%) patients did not access any mental health resource in the previous 12 months, and the most commonly accessed resource was hospital EDs (102, 76.1\%), with 24 $(23.5 \%)$ of these patients using the ED at least 6 times. Patients also accessed a variety of other mental health resources, with $28(20.9 \%)$ seeing their family physician, 20 (14.9\%) seeing their psychiatrist/ psychologist, and $61(45.5 \%)$ seeing both in the previous 12 months. Only $6(5.9 \%)$ patients used the ED exclusively for a mental health related complaint. By comparison, respondents accessed other specific mental health resources such as crisis centres $(19,14.2 \%)$, helplines (34, 25.4\%), and peer-support groups $(24,17.9 \%)$ less often. Conclusion: These findings suggest that the ED is the most commonly used mental health resource for this population. However, these patients also frequently access family physicians and psychiatrists/psychologists, with community resources such as crisis centres, helplines, and peersupport being used less often. This suggests that lack of timely access to other mental health resources may be the primary motivation for accessing the ED.

Keywords: mental health, health care utilization, emergency department

\section{P120}

Clinical decision rule evidence ranking and use in clinical practice S. Upadhye, MD, MSc, A. Chorley, MD, N. Arora, BHSc, McMaster University, Hamilton, ON

Introduction: The 2007 SAEM Knowledge Translation consensus conference proposed areas of research in evidence-based clinical algorithms (EBCAs) using clinical decision rules (CDRs) and practice guidelines (CPGs). This project sought to explore the evidence awareness and utilization of various clinical decision rules (CDRs) in emergency medicine (EM) practice. This project sought to explore the evidence awareness and utilization of various clinical decision rules (CDRs) in emergency medicine (EM) practice. Methods: An international survey was administered via international EM organizations using modified Dillman methods. Categories of CDRs included imaging (7), infections (3), neurology (2), venous thromboembolism (VTE; 2), and other (2). Evidence levels were queried using JAMA User's Guide CDR rating scales (Levels I-IV). Confidence with supporting evidence and utilization of CDRs in practice were assessed on 7-point Likert scales. Correlation of evidence understanding and practice utilization were calculated using Spearman rho methods. Results: The majority of respondents $(\mathrm{n}=378)$ were Canadian $(72 \%),<15$ years full practice (64\%), residency trained (90\%), and trained in CDR methods $(73 \%)$. Evidence ratings were deemed high for all CDRs, although confidence in evidence ratings and practice utilization were more variable for specific rules. Comfort with evidence ranking and utilization in clinical practice were highly correlated $(\rho<0.0002)$. Conclusion: Among Canadian residency CDR trained physicians, evidence ranking is strongly correlated with use in self-reported clinical practice. There is insufficient data from non-Canadian respondents to draw firm correlations. Their remains opportunity to fully disseminate high quality CDRs and encourage incorporation into EBCA practice.

Keywords: clinical decision rules, knowledge translation, levels of evidence 